\title{
HERCULES SAXANUS.
}

Hercules Saxanus ist auf altären und votivsteinen in und bei den tuffsteinbritichen des zwischen Andernach und Remagen mulndenden Brohltals mehr als zwanzigmal, in den kalksteinbrtichen des Moseltals bei Norroy-sous-Prény, die unweit Pont$\grave{a}$-Mousson oberhalb Metz liegen, dreimal und in Trient und in Tivoli je einmal bezeugt. Der erste gelehrte, der sich tuber mehrere jener rheinischen Hercules-Saxanusdenkmäler und den tiburtinischen stein äusserte, war J. G. Keysler in seinen Antiquitates selectae septentrionales et celticae, Hannoverae 1720, $190 \mathrm{ff}$. $\mathrm{Zu}$ unserm erstaunen sehen wir, dass dieser vielgereiste und ebenso belesene, wie kritisch urteilende mann, der ubrigens auch meines wissens zuerst den christlichen grundcharakter der Voluspá und den starken einfluss des christentums auf die jüngere Edda richtig hervorhob (a. 0. s. 126), die verfuhrerischen abwege, die ein jahrhundert später J. Grimm und weiterhin Kern und Simrock bei der erklärung dieses gottes einschlugen, sicher erkannte und besonnen vermied, um selber den richtigen, aber noch von den neuesten forschern wider aufgegebenen weg zu verfolgen. Er erwog nämlich die drei möglichkeiten eines zusammenhangs des Saxanus erstens mit dem deutschen gotte Saxnot, ferner mit den deutschen personennamen Sahso, Saxunus, endlich mit dem nordischen gotte Dorr, dem besieger des felsriesen Hrungnir, also dieselben möglichkeiten, die später die drei erwähnten germanisten nach einander erwogen. Aber er beschämte auch die allerneuesten forscher, die Simrock folgen, Jordan, den bearbeiter der 3. auflage von Prellers Römischer mythologie, und R. Peter, der einen wertvollen artikel uber Hercules fur Roschers ausfubrliches lexicon geschrieben, denn er erklärte den Saxanus schlichtweg aus dem lateinischen als 
einen römischen felsengott oder -heros, dessen beiname an die unter dem sogenannten saxum des Aventin hausende Bona Dea Subsaxana erinnere. Die ganze noch nicht abgeschlossene geschichte der hin- und herschwankenden deutung des Hercules Saxanus ist also im grunde nur eine evolution der alten Keyslerschen zweifel und gedanken. Sie besteht aus lauter 'Keysleriana'. Grimm nämlich nahm eine verwantschaft des lat. saxum 'stein' und des deutschen sahs 'messer, kurzes schwert' an. Das deutsche wort habe fruher eine steinwaffe bedeutet, der Saxanus sei als ein mit einem steinschwert oder steinmesser bewaffneter gott der Germanen zu denken, identiseh mit dem Saxnot, d. h. schwertgenossen oder schwertfuhrer des altsächs. taufgelöbnisses, einem beinamen des kriegsgottes Tiu (D. myth. 1203 ff.). Nun kann die möglichkeit einer urverwantschaft des saxum und des sahs nicht bestritten werden, aber weder jenes, noch dieses wort ist in dem sinne 'steinwaffe' nachweisbar oder nur glaublich, und der gemeinsame ausgangspunkt der begriffe beider wörter scheint in einer ganz anderen sphäre zu liegen. Beide sind nach Ascoli 1 ) aus der im lat. sec-are 'schneiden' erbaltenen wurzel ska entsprungen, sodass saxum ursprunglich das scharfe, klippige gestein, sahs aber die schneidige waffe gewesen wäre. Der unwahrscheinlichkeit jener Grimmschen etymologie steht aber die unwahrscheinlichkeit der mythologischen combination kaum nach. Denn der göttername alts. Saxnôt, ags. Saxnéat kommt nur innerhalb des Sachsenstammes vor, der in der fruberen kaiserzeit, in der die Saxanusdenkmäler entstanden sind, nie am Mittelrhein oder gar an der Obermosel aufgetreten ist und auch nie den legionen, die diese denkmäler setzten, soldaten geliefert bat. Auch wäre es höchst auffällig, dass ein bewaffneter kriegsgott, wie ihn Grimm annimmt, nie in den doch noch viel häufigeren inschriften der römischen legionslager und auxiliarstationen, sondern immer nur in steinbrüchen, wie sich zeigen wird, geehrt worden wäre. Obgleich Grimm an der steinbedeutung des wortes sahs auch noch im wörterbuch s. v. fels festhielt, liess er doch später seine mythologische combination aus rilcksicht auf die mehr-

1) Kuhns zs. 16, 207. Fick, Vergleich. wb. 23, 252 setzt ein urwort *sakso 'steinschneide' an. 
fachen ausserdeutschen denkmäler dieses Hercules fallen 1 ); dennoch spukte sie weiter, wenn auch in veränderter, der zweiten Keyslerschen form. Denn Kern holte nun zur rettung des germanischen Saxanus die alten namen Saxani, Saxini aus einer Zutphener urkunde des 9. jhs. hervor, denen man allerdings die viel ältere rheinische Sacsena und manche andere ähnliche altdeutsche namen beifügen könnte. ${ }^{2}$ ) Aber keine Herculesinschrift deutet eine derartige schwächung oder kürzung des suffixvocals an, wie sie diese namen voraussetzen. Auch war es nicht deutsche art, gōtterbeinamen als unzusammengesetzte personennamen zu verwenden, freilich wol als fluche. Allein der von Kern ebenfalls herangezogene fluch ui saksn!, den der Oberpfälzer neben ui dunner! ui straul (d. i. blitz)! gebraucht, ist schon von Schmeller ${ }^{3}$ ) als ein verkapptes ui sakra! erkannt worden. Endlich bemächtigte sich Simrock ${ }^{4}$ ) des dritten Keyslerschen gedankens, indem er sich auf die ältere interpretatio romana des germanischen Thunar durch Hercules, wie wir sie bei Tacitus finden, stlutzte. Im Brobltal babe der deutsche donnergott als felsenspalter, lat. Saxanus, den steinhauern geholfen, wie der nordische pórr den felsgrund zu baulichem grunde bearbeitet habe. Allerdings zerschmettert bórr im nordischen mythus die felsen und bringt den felsriesen Hrungnir zu falle. Aber dass er mit dieser tat dem acker habe nutzen wollen, davon hat Uhlands ${ }^{5}$ ) deutung dieses mythus schwerlich uberzeugt. Nicht durch felszersehmetterung, sondern durch warmen gewitterregen segnet porr die fluren, deren schutzgott er allerdings ist.6) Auch darf die nordische Dorrsvorstellung nicht ohne weiteres von dem fast ganz aus felsgestein aufgebauten Norwegen auf das anders geartete Deutschland, noch vom ackerbau auf den steinbruch tibertragen werden. Aber dies auch zugegeben, die Deutschen ktimmerten sich vor der ankunft der Römer nicht im geringsten ums steinbrechen; weil

1) Grimm, D. myth. 2302.

2) Revue celtique 2,158 f. Brambach, Corp. inscript. Rhenan. nr. 194. Forstemann, Altdeutsches namenbuch 1, 1066.

3) Schmeller, Bayr. wb. 3, 193.

4) Simrock, Handbuch d. d. mythol. ${ }^{5} 244 \mathrm{ff}$.

5) Uhland, Thor s. 43.

B) E. H. Meyer, Germ. wythol. $§ 290.267$. 
sie ihre heiligtlumer und burgen, häuser und grabmäler erwiesener massen aus erde, lehm, holz und etwa noch aus findlingsblöcken bauten. Sie konnten also gar nicht auf die idee eines steinbruchsgottes kommen. Einen solchen lehrten ihnen erst mit dem betreffenden handwerk die besten steinbrecher der welt, die Römer. Ueber die frage, ob denn auch nur romanisierte Germanen im Brohltal als steinbrecher, ob Germanen überbaupt nur als bewobner oder soldaten in diesem tal oder dem der Mosel oder gar bei Trient oder Tivoli, denkbar seien, mit andern worten uber die wichtige dedicantenfrage gieng Simrock trotz den durch die inschriften reichlich gebotenen anhaltspunkten schweigend hinweg. Dennoch stimmten ihm Jordan und der neueste Herculesmytholog R. Peter ${ }^{1}$ ) bei. Ja dieser und sein germanistischer gewährsmann Jaekel ${ }^{2}$ ) fielen in eine abart des etymologischen irrtums J. Grimms zurttck, indem sie in Saxanus einen germanischen Sahsan 'felsenbeherscher' sahen, ein wort, das aus sahs in der unerwiesenen bedeutung 'fels' und dem nomina agentis bildenden suffix -ana gebildet sei.

Glucklicher weise durfen wir gegenuber diesem unerfreulichen gegenwärtigen stande der Hercules-Saxanusfrage anerkennen, dass mebrere ältere classische philologen und archäologen der gesunden weisung Keyslers gefolgt sind und den gott als einen herrn des felsgesteins und der steinhauer römischen ursprungs auffassten. Aber auch in den hauptarbeiten dieser richtung, in Freudenbergs 'Das denkmal des Hercules Saxanus im Brohltal 1862' und P.-Charles Roberts 'Inscriptions laissées dans une carrière de la Haute Moselle par des légions romaines 1884' (in den Mélanges Graux 1884, s. 329) sind zwar manche einzelheiten der dedicantenfrage erledigt worden, allein ihre untersuchung hat nicht dazu gefuhrt, die denkmäler in ibrer zeitlichen reihenfolge, in ihrem zusammenhange unter sich und mit andern denkmälern und endlich in ihrem zusammenhang mit der grossen zeitgeschichte zu würdigen. Erst bei solcher behandlung werden sie zu unverächtlichen urkunden jener epoche der widerherstellung, in der die flavischen kaiser das römische reich aus blutigen bürgerkriegen und aufständen

1) Preller, Röm. mythol. 23, 297. Roscher, Ausführl. lex. 1, $3014 \mathrm{ff}$.

2) Vgl. Zs. fdph. 23, 138. 
heraushoben und mit ihren nachfolgern, Trajan und Hadrian, namentlich auch durch grossartige bauten und einschneidende heeresreformen von neuem befestigten. Zugleich aber nehmen sie als solche auch noch den letzten germanischen schimmer vom Hercules Saxanus hinweg.

Die Hercules-Saxanusdenkmäler des Brohl- und des Moseltals zerfallen nach ihrem alter in drei hauptgruppen, in I. eine vorflavische, II. eine vespasianische und III. eine trajanische. Die 1. und 3. gruppe besteht nur aus Brohler, die 2. aus Norroy- und Brohlsteinen.

I. Die vorflaviscbe gruppe. Ihre drei denkmäler sind gesetzt, zwei von der 15. und eine von der 16. niedergermanischen legion. Das erste, das auffälliger weise die legionshezeichnung an die spitze der dedication stellt, lautet:

1. LEG XV S. XANO POSVIT M. STATILIVS .. L. M. P. Brambach no. 685 : posuii statt posuit. Freudenberg no. 17.

2. HERCVIi SAXANO C. MEtTIVS SENECA LEg XV ET VEXILLARI LEG EIVSDEM V. S. L. M. Bonner jahrb. 50-51, $192 \mathrm{ff}$.

3. I. O. M. ET SAXSANO L. IVLIVS CIASSICVS .. LEG XVI et VEXILLARI. Brambach no. 65i. Freudenberg no. 14: Saxano statt Saxsano.

Da díe beiden niedergermanischen legionen, von denen die 16. den'aufständischen Batavern im jahre $70 \mathrm{n}$. Chr. in Neuss, die 15. bald darauf in Castra vetera sich ergeben hatte, wegen dieses treubruchs von Vespasian in diesem jahre aufgelöst wurden, so mtissen die obigen drei steine in die vorflavische zeit gesetzt werden. Die 16. legion kam wahrscheinlich in folge der durch die britannische expedition unter kaiser Claudius im jahre 43 notwendig gewordenen truppenverlegung aus Ober, nach Niedergermanien, aus dem ausschliesslicb, wie sich weiter unten zeigen wird, mannschaften fur die Brobler steinbruchsarbeiten herangezogen wurden. Die 15. legion wurde uberhaupt erst von demselben kaiser um dieselbe zeit geschaffen. ${ }^{1}$ ) Die dadurch auf die jahre $43-70$ begrenzte entstehungszeit der steine wird leider durch die officiersnamen, die keinen chronologischen anhalt gewähren, nicht noch enger umschrieben. Zwar ruft einem der Lucius Julius Classicus von no. 3 den vornehmen reichen Gallier Julius Classicus ins gedächtnis, der im jahre

1) Brambach a. a. o. s. X. XIII. Marquardt, Rüm. staatsverwaltung $2^{2}$, 446. 448. 
69 praefectus der damals berühmten Trierschen reiterei war und im jahre 70 auf dem zuge des römischen oberbefehlshabers Vocula gegen den Batarer Civilis zwischen Neuss und Vetera das römische heer treulos verliess, um das neue gallische reich zu proclamieren. Er zwạg die infolgedessen von Vocula nach Neuss zurickgefuhrten legionen zur capitulation und zum schwur pro imperio Galliarum, und grade die 16. legion erhielt nun den befehl, von Neuss über Bonn, wo sich noch eine andre ebenfalls abgefallene legion (die 1.?) ihr zugesellte, nach Trier abzumarschieren, Tac. Hist. 2, 14. 4,55 ff. 62. Man könnte weiter vermuten, dass sie auf diesem marsch von dem im siegesrausch völlig sorglosen Classicus, Hist. 4, 70, aus irgend welchem grundo angehalten und mit steinbruchsarbeiten beschäftigt worden sei, wie sie vorher in Neuss eifrig ziegel gebrannt hatte.1) Aber nach Zangemeisters gutiger mitteilung ist auf dem von ihm selbst copierten steine zwischen Classicus und Leg höchstens für das centuriozeichen 0 raum, das auf den damaligen praefectus alae nicht passte. Aber auch an eine fruhere rangstufe des vornebmen praefectus darf man wol nicht denken, da das avancement eines legionscenturio zum praefectus alae in dieser periode kaum möglich war. ${ }^{2}$ ) Es scheint demnach eine zufällige namengleichheit vorzuliegen, die noch dazu fur das praenomen nicht nachgewiesen werden kann, weil es von dem aufständischen nicht uberliefert ist. Wir mussen also die steine der claudisch-neronischen epoche zuweisen und zunächst als denkmäler römischer legionssoldaten anerkennen. Eine ganze legion, wie es nach nr. 1 den anschein hat, oder die vexillarii einer solchen, $d . b$. die legionsdetachements, die von einem centurio bez. decurio oder tribunus militum in steinbrichen und bergwerken commandiert zu werden pflegten ${ }^{3}$ ), setzten bei ihrer schweren arbeit im steinbruch dem Hercules Saxanus, um seinen schutz zu erflehen oder ihm ihren dank dafür auszusprechen, einen votivstein oder eine ara. Der dritte schloss den mächtigen vater des gottes, den Jupiter Optimus Maximus, mit in ihr votum ein. Ist darin irgend etwas unrömisches zu verspüren? etwas

1) Bonner jahrb. 84, 1887, 261.

2) Doch vgl. Marquardt a. a. o. 2, 378.

3) Marquardt a. a. o. 2, 265. 
germanisches? Allerdings spielte in der damaligen Rbeinarmee auch der miles peregrinus et externus eine bemerkbare rolle, Tac. Hist. 2, 21, jedoch uberwogen in der neronischen zeit die italischen, raetischen und gallischen elemente in den legionen das germanische weitaus und um so mehr, als unter den früheren kaisern die beiden Germanien, die ausserordentlich viel mannschaft für die auxilien stellen mussten, kaum noch leute an die legionen abgeben konnten. ${ }^{1}$ ) Ja, der aufstand des Civilis wurzelte grade in der eifersucht der batavischen hilfstruppe auf die bevorzugten, aus ungermanischen fremdlingen zusammengesetzten legionen. Eher könnte man die Brohler steine auf die damals viel stärker in diesen vertretenen Gallier zurickfuhren. Denn. auch die anwohner des steinigen Brohltals, das vor der Römerherschaft wahrscheinlich kaum beachtet dalag, waren im 1. jahrhundert schwerlich echte Germanen, sondern entweder mit romanisierten Ubiern gemischte oder reine treverische Kelten, da der keltisch benannte Vinxt- oder Pfingstbach, der später als grenze Ober- und Niedergermaniens galt, unterhalb der Brohl die grenze zwischen den Ubiern und Treverern gebildet haben wird.2) Deutsche gelangten erst weit später in den besitz dieser strecke des linken Rheinufers, die damals völlig unter dem einfluss des stark romanisierten keltischen Moseltals stand, und man könnte die annahme gallischen ursprungs auch noch dadurch bestätigt finden, dass grade im oberlauf dieses Moseltals Hercules Saxanus ungefähr um dieselbe zeit ebenfalls in steinbrlichen verehrt wurde. Dieser ansicht waren Kaemmel und ihm folgend auch ich ${ }^{3}$ ), aber sie ist irrig, wie die nähere betrachtung eben dieses Hercules von der Mosel alsbald ergeben wird.

II. Die vespasianische gruppe zerfăllt a) in die gruppe von Norroy und b) in die von Brohl.

a) Die gruppe von Norroy. In den kalksteinbrichen von Norroy oberhalb Metz sind gefunden worden ein votivstein und zwei altäre:

1) Mommsen im Hermes 19, 15. 19. 21. j5.

2) Th. Bergk, Bonn. jahrb. 57, 31 ff. Esser, Beitrïge zur 'gallo-keltischen namenkunde 1, 73.

3) Kaemmel, Anfänge des deutschen lebens in Oesterreicn s. 38. E. H. Meyer, Germ. mythol. 202. 
4. HERCVLI. SAXSANO. VEXILLARI. Le. XXI. RA. ET AVXILIA. EORUM COHORTES. V QVI. SVNT. SVB. L. POMPEIO. SECVNDO. LE. XXI. V. S. L. M. Freudenberg no. 28. Robert a. a. 0. 330.

5. I. O. M. ET HERCVLI. SAXA SACRVM P. SALPIDIVS CLEMENS. LEG VIII AVG. CVM MILi LEG. EIVS V. S. L. L. M. Freudenberg no. 26. Robert a. a. 0.334 .

6. HERCVLI. SAXSANO ET IMP VISPASIANO AVG. ET. TITO IMP ET DOMITIANO CAESARI $M$. VIBIVS MARTIALIS LEG $\cdot X \cdot G E M$. ET COMMILITONES VEXILLI. LEG EIVSD QVI. SVNT SVB CVRA EIVS V. S. L. M. Caylus-Freudenberg no. 27: Vespasiano statt Vispasiano. Robert a. a. o. s. 332.

Diese drei lothringischen denkmäler sind von drei verschiedenen truppenkörpern, der 8. und 10. legion und den vexillarii der 21. legion, errichtet. Jene beiden legionen kamen zuerst im jahre 70, die eine aus Moesien uber Italien, die andre aus Spanien, nach der provinz Belgica, in deren gebiet die steinbrïche von Norroy lagen, vielleicht auch damals zuerst die obergermanische 21. Fur diese wird die zeit ihrer dortigen arbeit noch etwas näher bestimmt durch ihre auflösung, mit der sie wegen ihrer teilnahme am militäraufstand des Saturninus im jahre 88/89 bestraft wurde. ${ }^{1}$ ) Noch enger wird dieser zeitraum von 70/89 begrenzt fur die 10. legion, denn Titus wurde zum ersten male als imperator im jahre 71 ausgerufen und Vespasian starb im jahre 79. Demselben zeitraum werden aber auch die steine der 8. und der vexillarier der 21. legion angehören und wahrscheinlich alle drei dem anfange dieses jahrzehnts, den jahren 70 und 71. Denn in diesen jahren waren diese drei truppenkörper nachweisbar im gebiete der Mediomatriker, der Metzer, in dem Norroy liegt, zu kriegszwecken und im sudlich angrenzenden

1) Hermes 19, 15. Asbach, Westd. zs. 3, 10. 10, 122. Marquardt, Rüm. staatsverwalt. $2^{2}, 450$. Schiller a. a. o. 1,581 vermutet, die 21. legion sei schon im sarmatischen krieg $86 \mathrm{zu}$ grunde gegangen. Die paläographischen gründe, die Robert a. a. 0. 331 für die annahme eines hơheren alters der inschrift der 21. legion anführt, sind nicht stichhaltig, deun die trennungszeichen gleichen nicht denen der von ihm citierten inschrift des kenotaphs eines centurionen aus dem heere des Varus (CIRh. nr. 209). Die buchstabenform ist durchans nicht besonders altertïmlich und die schreibart Saxsanus kommt mehrfach auch noch unter Trajan vor. Mehr gewicht hat Zangemeisters freundliche briefmitteilung, dass die abkïrzungen $R A$ (pax) und $L E$ (gio) statt $R A P$. und $L E G$. sich grade in den ältesten inschriften der Rheinlande finden. Doch s. oben. 
gebiete der Lingonen (Langres) zu anderweitigen bauarbeiten, namentlich zum ziegelbrennen, vereinigt. Grade die drei genannten legionen nämlich kamen, die 8. und 21. aus Italien, die 10. aus Spanien im jahre 70 bez. 71 in den genannten ländern der Germania superior und der Belgica zusammen, bez. dicht hinter einander her, um mit der ubrigen grossen armee des vespasianischen feldherrn Cerialis nordwärts gegen die aufständischen Trierer und Bataver vorzurticken. Diese truppenteile scheinen aber nicht zum schlagen gekommen, sondern noch bis uber den zeitpunkt der unterdruckung des aufstands, die schon im jahre 70 stattfand, in der Belgica stehen geblieben und mit bauarbeiten beschäftigt worden zu sein. Hier kommt uns ein bei Mirebeau-sur-Bèze gemachter fund zu statten, der bisher nicht mit unsern Herculesdenkmälern in verbindung gebracht worden ist. Mirebeau liegt slidlich vom plateau von Langres 22 kilometer nordöstlich von Dijon unweit der alten heerstrasse, die von Lyon tiber Metz, also auch an Norroy vorbei, nach Trier lief. Hier hat man vor etwa 10 jahren einen 80 meter langen in den felsen gehauenen aquaeduct, mehrere inschriftsteine und insbesondere zahlreiche einfach bezeichnete legionsziegel, aber auch solche entdeckt, die nach ihrem stempel von combinierten detachements mehrerer legionen angefertigt sind. Einer dieser ziegel trägt den stempel der vexill. legionum $I$. VIII. XI. XIV. XXI, andere tragen andere gemeinsame stempel, so einer auch die combinierung der vexilla der II. und VIII. Mowat und Mommsen schliessen aus dieser funfzahl oder gar sechszahl mit recht, dass eine derartige baumannschaft nur aus einer so ausserordentlichen truppenconcentrierung habe hervorgehen können, wie sie im jahre 70 in Obergermanien unter Vespasians feldherrn Cerialis $\mathrm{zu}$ stande kam und wie sie uns auch die combinierte steinbruchsarbeiterschaft von Norroy erklårt hat. In der tat setzten die sechs legionen, deren detachements wir in Mirebeau finden, mit zwei andern hier nicht genannten die grosse armee Vespasians zusammen. Aus Italien trafen die II. Adjutrix, die VIII., XI., XXI. und wahrscheinlich die XIII. Uber die nordwestlichen alpenpassse, die I. und VI. aus Spanien und die XIV. aus Britannien ein. Alle wanten sich zunächst nordwărts gegen den Oberrhein, nur die 14. marschierte von der nordseekliste ostwărts gegen den Nieder- 
rhein. Funf von jenen legionen, aber auch diese 14., gaben abteilungen $a b$; die sich im gebiet der Lingonen vereinigten, wo die strasse von Lugdunum über Metz und Trier nach dem Rhein einerseits eine heerstrasse vom Genfersee aufnahm, andrerseits eine andre nach der nordsee abzweigte.1) Wenn die aus dem gros der legionen gebildete hauptarmee Vespasians ibren ersten kräftigen stoss von Mainz ber uber die Moselstadt Rigodulum, -dunum ${ }^{2}$ ) gegen das aufständische Trier fubrte, wie Tacitus berichtet, so lernen wir aus diesen ziegeln und den steinbruchsdenkmälern von Norroy eine von ihm unerwähnte, aus detachements von wenigstens sechs jener legionen combinierte seitenarmee kennen, die offenbar dazu bestimmt war, die zu den gallischen Treverern abgefallenen Lingonen im gebiete von Langres zu züchtigen, an jener wichtigen heerstrasse die zerstörten oder auch neue feste lager aufufuhren und von süden her uber Metz ebenfalls gegen Trier vorzudringen. Da nun jene von Classicus nach Trier geschickten untergermanischen legionen (o. s. 111) zu den romfreundlichen Metzern ubergiengen und Trier bald dem angriffe jenes hauptheers erlag, so blieb ein teil der nebenarmee, den man zum kriege nicht mehr brauchte, oberhalb Metz stehen, nämlich die detachements der 8. und 21. legion, um fur befestigungszwecke in den kalksteinbruchen von Norroy zu arbeiten. Ihnen schloss sich hier noch eine dritte spanische legion, die 10., an, die auch nach Mommsens vermutung erst später marschbefehl erhalten hatte. ${ }^{3}$ ) Sie war es denn auch, die nach dem inzwischen errungenen vollen siege des kaisers uber Civilis auf ihrem altar in dem steinbruch die huldigung fur den Hercules Saxanus mit der fur Vespasianus und seine söhne vereinte (s. no. 6). So stellt sich die steinbruchsarbeit im gebiet der Mediomatriker als das zweite stadium der bautătigkeit der gegen norden vorrickenden vespasianischen legionsabteilungen dar, deren erstes stadium im sudlichen nachbargebiet der Lingonen lag. In éinen blick zusammengefasst, lehren uns die drei ältesten Brobl-, wie diese drei Norroydenkmäler, dass die

1) Mommsen im Hermes 19, 437. Dazu vgl. Tac. Hist. 4, 68. 76. 5,14. Strabon IV c 208. Bonner jahrb. 57, 52.

2) = Regadonum a. 804. Beyer, Mittelrhein. urkundenb. 1, 46; vgl. Esser, Beitr. z. gallo-keltischen namenkunde 1,7.

3) Hermes 19,440 anm. 1. 
römischen soldaten in den 50 er -70 er jahren des 1 . christlichen jahrhunderts, mōgen sie germanischen oder keltischen localeinflussen ausgesetzt oder entruckt, in einer nieder- oder obergermanischen, moesischen oder spanischen legion dienen, in den steinbrtichen einen und denselben gott verehrt haben. Und es wird immer deutlicher, dass dieser römische soldatengott mit römischem namen und beinamen niemand anders ist als der seit jahrhunderten in Italien verehrte ausdauernde heros gefahrvoller kämpfe und mühseliger arbeiten, der hier in echt römischem sinne zu einem Hercules Saxanus, zum schutzgott der mubseligen soldatenarbeit in den steinbrüchen, weitergebildet erscheint.

b) Zur gruppe von Brohl, soweit sie in Vespasians regierung fällt, gehören wahrscheinlich zunächst folgende zwei steine :

7. HERCVLI-SAXSANO SACRVM. IVLIVS VICTOR O PRO SE ET COMI. LITO. NES. S. S. (die zwei nächsten zeilen sind undeutlich) LEG. AVGV (?) SLM. Bramb. no. 663. Freudenb. no. 18.

8. H .... LI SAXANO LLICINIVS FESTVS O LEG XXI rAP-ET MILITES IeG. EIVSDEM VLS. Bramb. no. 656. Freudenb. no. 6. Catalog d. Bonner mus. no. 23.

Wenn wirklich in no. 7 eine legio Augusta genannt ist, 80 wird nicht die legio II Augusta, die seit kaiser Claudius in Britannien stand, sondern die schon in Mirebeau und Norroy zu bauzwecken verwendete legio VIII Augusta gemeint sein; dies ist um so wahrscheinlicher, als wir nach no. 8 auch eine ihrer gefährtinnen von Mirebeau und Norroy, die 21. Rapax, in Brobl widerfinden. Sie oder ibre detachements waren also beide wåhrend des krieges mit Civilis oder wahrscheinlicher nach Vespasians sieg nach Untergermanien oder an dessen grenze gerllckt, um hier durch steinbruchsarbeiten die nach den verwhistungen des Civilis notwendig gewordenen neubauten vorzubereiten. Eine spätere zeit als die vespasianische scheint ausgeschlossen, da Domitian in Ober-, nicht in Untergermanien baute und des Brohler materials nicht bedurfte, die 21. legion jedenfalls im jahre 88-89 aufgelöst wurde (s. 0. 8. 113) und die 8. später, z. b. im jahre 90 , nachdem sie eine obergermanische legion geworden, in viel sudlicheren strichen, in Aquitanien und in der Narbonensis, ziegel brannte. ${ }^{1}$ )

1) Hermes 19, 498. 
Möglicherweise schliessen sich diesen steinen die halb zerstörten an :

8a. ... AC G. XXI. ENIARIS I. M. Bramb. no. 669 und

8 b. HERCVLI SAXAN. Bramb. no. 668 .

III. Die dritte hauptgruppe ist um ein paar jahrzebnte junger und wenigstens zum grössten teil unter Trajan zu setzen:

9. IMP. CAES. TRA AV GER III ... IVL .... HERCVLI ..... TVBIC ... Bramb. no. 667. Freudenb. no. 22.

Dieser stein wurde gewidmet während des dritten consulats des Trajanus Augustus Germanicus d.i. im jahre 100.1)

Im jahre 101, in welchem die Arvalen fur die ruckkehr Trajans vom ersten dacischen feldzuge unter andern gottheiten auch dem Hercules Victor gelubde taten ${ }^{2}$ ), oder in einem der nächstfolgenden jahre wurden dem Hercules Saxanus im Brohltal verschiedene denkmäler geweiht, darunter ein vornehmeres, das freilich seinen beinamen nicht nennt, aber ohne zweifel ihn meint. Denn auf der geglătteten, mit. schwachem kalkgrund belegten, oben giebelförmig zulaufenden partie einer gen osten gekehrten felswand sind funf nischen dargestellt. In der mittleren, deren bogen und pfosten eine oben mit einer gelblichen kugel versehene spitzsăule tragen, ist ein Herculesaltar in den fels gehauen, wăhrend die kleineren seitennischen rotgemalte flammende leuchter umschliessen und oben entweder mit einer mondsichel oder mit sonnenstrablen verziert sind. Freudenberg a. $0.25 \mathrm{ff}$. möchte diese fremdartige ausstattung des Herculesaltars lieber auf nachahmung der cultusformen des halb phönicischen Hercules Gaditanus, als auf vermischung des römischen Herculescultus mit dem cultus des Mithra, des Deus oder Sol Invictus, zurluckfubren. Beides muss ich dahin gestellt sein lassen, möchte aber darauf aufmerksam machen, dass eine dedication zuerst die capitolinischen gottheiten Jupiter, Juno und Minerva nennt, um sich darauf dem Soli Mithrae Herculi Marti Mercurio

1) Klein, Fasti consulares s. 52. Nach einer alten erzählung widmete in Rom ein tibicen, der einen seeräuberangriff zurïckgeschlagen hatte, dem Hercules Victor einen tempel, der das älteste denkmal des Hercules Victor gewesen zu sein scheint, Preller, Röm. mythol. $2^{3}, 294$.

2) CIL. 6, s. 530, vgl. Henzen, Acta fratr. Arval. $123 \mathrm{ff}$. 
Genio loci dis deabusque omnibus zuzuwenden.1) Noch näher kommen unserm nicht nur mit dem symbol der sonne, sondern auch mit dem des monds verzierten Herculesdenkmal die in Rom gefundenen weihinschriften der Equites singulares, von denen eine den Juppiter O. M., Sol Divinus, Mars, Mercur, Hercules u. s. w., die nächste Juppiter, Juno, Sol, Luna, Hercules, Minerva, Mars u. s. w. zusammenstellt. ${ }^{2}$ ) Für unsern zweck ist viel wichtiger als die malerisch-architektonische ausstattung die inschrift jenes altars, welche lautet:

10. T H. ER L. VI. VI. PF. L. X. GP. F. L. XXII. PRP ET. AL. CO. CL. Q. S. Q. ACVT SU. CV. M. I COSSVTI. X. L VI VIC PF, Bramb. no. 660. Freudenberg 8. 16 liest za anfang: $I$ statt $T$, d. h. Invicto, und weiterhin Cossuli 0. $\mathrm{Zu}$ Invicto Herculi statt Herculi Invicto vergl. man $I . M$. = Invicto Milhrae CIRh. no. 285. 527. Das ganze bedeutet: (Invicto?) Herculi legio $V I$ Victrix pia fidelis, legio $X$ Gemina pia fidelis, legio XXII Primigenia pia et alae, cohortes, classis, qui (sunt) sub Q. Acutio sub cura M. Julii Cossutii centurionis? legionis VI Victricis piae fidelis. Aehnlich ist

11. IOM ET HER SAX VEXIL L VI. VICPF LXG PF ET AL CO CLAG PF Q S 0 ACVT SV CU M. IVL COSSVTI L Vị VIC PF. Bramb. no. 662. Marquardt. Röm. staatsverwaltung 22, 506. Freudenberg, Bonner jahrb. 38, 84. Statt der legionen in no. 10 werden hier nur legionsvexillarii genannt und die 22. legion fehlt. Die ubrigen mannschaften sind auch hier vertreten, doch ist die classis zubenannt $G, d$. h. Germanica. Widerum ähnlich lautet:

12. HERCV SA VEXILLARI LI MF L VI VICT. L X GP ET AL CO CL QS O ACVT SV CV $M$ IVLI COSSVTI L VI VIC PI. Bramb. no. 680. Freudenberg no. 2. Hier weihen die vexillarier dreier legionen, der 6., der 10. und, nicht der 22., sondern der 1. Minervia.

Alle diese vier legionen unterstanden dem Q. Acutius Nerva und arbeiteten im Brohltal unter der aufsicht eines centurio. Mit diesem namen aber gewinnen wir einen neuen festen zeitpunkt, denn Q. Acutius Nerva war consul designatus im jahre 100 n. Chr. und muss nach diesem jahre als legatus Untergermanien verwaltet haben. ${ }^{3}$ ) Die drei denkmäler zeigen uns ferner zuerst den durchgreifenden legionswechsel, der nach der unterdrickung des Bataveraufstandes in Germanien eintrat. Die alten vier untergermanischen legionen, von denen wir die 15. und die 16. friber in der Brohl tätig sahen, sind verschwunden und von den vier obergermanischen ist jetzt nur

1) CIL. 8, 4578.

2) Henzen in Ann. d. inst. 57, 1885, no. 22. 23.

3) Klein, Fasti consulares 53. Mommsen, Röm. gesch. 5, 133. 
noch die 22. geblieben, die trotz ihrer teilnahme an jenem aufruhr von der auflösung verschont worden war und darauf nach Untergermanien kam.1) Zu ihr gesellten sich dann zwei spanische legionen, die 10. Gemina, die uns schon von Norroy her bekannt ist, und die 6., die Vespasian ebenfalls im jahre 70 aus Spanien hieher berief (0.8.114f.). Die 1. Minervia endlich wurde erst von kaiser Domitian, dessen lieblingsgottheit Minerva war, ums jahr 90 errichtet, um jene cassierte 21. zu ersetzen. Die vier legionen bildeten seit Vespasian bez. Domitian das untergermanische heer.

Aber nicht nur im verein mit andern legionen, wie auf den Acutiussteinen, sondern auch die einzelne legion fur sich weihte Hercules-Saxanusdenkmäler. Nur von der ersten Minervia ist uns kein derartiges erhalten. Die eifrigsten dedicanten waren die Spanier. Die 6., die an allen drei Acutiusinschriften beteiligt war, widmete unserm gotte auch noch:

13. I. HERC. SAXAN SACR. IVLIVS VERECVNDVS . . LEG VI VI. . . IVAR .. AN . . Das weitere fehlt. Bramb. no. 664. Freudenberg no. 19. Man darf ihr anreihen als no.

14. HERC SAXSA SACR IVLIVS VERECVNd CENTVRIO COH .... VAR (ci) AN (orum) EX VOTO. Bonner jahrb. 81, 112.' Die aus oberpannonischen Varciani oder aus Varoduli (s. u. no. 18 b) zusammengesetzte zusammengesetzte oohorte scheint eine hilfstruppe der 6. legion gewesen zu sein, da sie unter dem commando eines ihrer centurionen stand, wie der vergleich mit no. 13 ergibt.

Noch zwei andere Herculeswidmungen der 6. legion schalten wir hier ein, obgleich sie dẹn gott nicht als Saxanus, sondern als Invictus bezeichnen:

14 a. HERCVLI. INVICTO. SACR ...... RENTIVS. BASS. O. LEG VI VICT ET VEXILLAR LEG. EIVSDEM. Brambach no. 654. Freudenberg no. 12, bewahrt im Wiesbadener museum. Fast gleich lautet:

14 b. HERCVLI INVICTO SACRVM C. TERENTIVS BASSVS O LEG. VI VICTRICIS ET VEXILATIO LE EI. Freudenberg no. 11. Bonner jabrb. 84, 73, bewahrt in Brohl. Noch rühriger scheint die schon in Norroy beschäftigte 10. legio Gemina gewesen zu sein:

15. I. O. M. HER SAX SEX DONNIVS VINDEX O LEG X G. P. FD. ET COMMILITONES VS. L. M. Bramb. no. 651. Freudenberg no. 9: Ses statt Sex. Catalog d. Bonner mus. no. 22.

1) Mommsen a. a. o. 121. 130 . 
16. I. O. M. ... RC ... XA .. C NIGIDIVS APONIVS. O LEG X. G. PF Et COMmILITONES LEG. EIVSDEM VV S. L. M. Bramb. no.652. Freudenberg no. 7.

17. HERCVLI SAXSANO $Q$ MANLIVS PRISCVS O LEG $X$ GEMINA ET COMMILITONES V S I. Bramb. no. 655. Freudenberg no. 8. Catal. d. Bonner mus. no. 21.

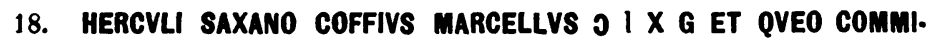
LItoNES V. S. L. M. nach Nimwegen wol aus Brohl verbracht. Bramb. no. 679. Frendenberg no. 3: Coelius statt Coffius.

Dazu stelle ich den stein eines trompeters dieser legion, der freilich nicht dem Hercules Saxanus, sondern dem H. Barbatus in Brohl seine verehrung bezeugte. Anf den zahlreichen sog. viergöttersteinen Obergermaniens wird Hercules immer mit einem vollbart dargestellt. ${ }^{1}$ )

18 a. HERCVLI BARBATO SACRVM M. HELLIVS SECVNDVS TVBICEN LEG. X. G. P. F V. S. L. M. Bramb. no. 653 .

Die sieben denkmäler der zwei spanischen legionen VI und $X$, no. $13-18 \mathrm{a}$, stammen wahrscheinlich wie die Acutiusdenkmäler no. 10-12 und der trompeterstein aus der regierungszeit Trajans, sind wenigstens nicht viel später anzusetzen. Denn die 6. legion schickte Hadrian vom Niederrhein nach York, und im jahre 122 arbeitete sie am Hadrianswall.2) Die 10. gieng unter Hadrian oder Antoninus Pius nach Pannonien, und einzelne ihrer detachements nahmen am kriege gegen die aufstandischen juden 132-135 teil.3) Auch tritt auf keinem denkmal die erst von Trajan errichtete 30. legio Victrix auf, obgleich der Brobler steinbruch doch wahrscheinlich grade für ihr winterlager, die Castra Trajana bei Vetera, wie unten bemerkt werden wird, das material liefern musste.

Im verbande mit einer dieser spanischen legionen stand wol die 2. cohorte der Asturier:

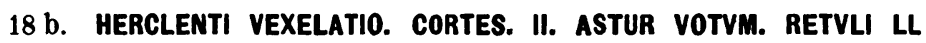
LI (?)B. Bramb. no. 666. Freudenberg no. 21 : cortis statt cortes, was beim bekannten wechsel von $i$ und $e$ in solchen endungen nicht von belang ist. HERCLIN'II bei Bramb. no. 315 seitens einer coh. II Var (dulorum? Bramb. oder -cianorum) s. o. no. 14 und DEO HERCVLEN'II bei Bremenium (Ribchester) CIL. 7, no. 1032. Die 2. asturische cohorte ist unter Hadrian in Britannien stationiert, wie auch die 6. legion (s. o.) und andere asturische truppen.4) Dazu kommt no.

1) Westdeutsche zs. 10, 304.

2) Schiller a. a. 0. 1, 157. 608.

3) Schiller a. 2. 0. 1,614. 642 .

4) Freudenberg a. a. o. 19 ff. Hübner im Hermes 16, 578. 
19. hercli. saxano. gemellvs. IMaginif COH ... astvavm. Pod et VEXIL. SCOH EIVSDEM V. S. L. L. M. Brambach no. 678. Freudenberg no. 5.

Die dritte auf den Acutiussteinen vertretene legion, die XXII. Primigenia, weiht folgende denkmäler:

20. hercVli. saxano. SacrVm. VeXsillatio leg. XXII PR. QVI. SVNT. SVB CVRA. K. APRILI. O M. Brambach no. 672. Freudenberg no. 4.

21. HERCVLI. SAXSANO. SACRVM. C. SVLPICIVS. MATVRVS. O LEG. XXII PR. P. F. ET. COMMILITONES. LEG. EIVSDEM. QVI. SVB EO. SVNT V. S. L M. Brambach no. 674. Freudenberg no. 1.

Wahrscheinlich gehört hierhin wegen seines fundorts in den Brohler steinbrichen auch:

21 a. . . . . . NVS. O LEG XXII P. P. F. ET. COMMILIT QVI CVM EO SVNt V. S. L. M. Brambach no. 671. Freudenberg no. 13. Catalog des Bonner mus. no. 25.

Die von Vespasian aus Ober- nach Untergermanien versetzte 22. legion schickte Hadrian wie die 6. (0. s. 120) nach Britannien. Erst um das jabr 170 begegnen wir ihr wider in Obergermanien, von wo aus sie erst um die mitte des 3. jhs. nach Afrika kam.1) Auch diese inschriftsteine werden daher am besten in die zeit Trajans gesetzt, in welcher die 22. legion ja nach nr. 10 im Brohler steinbruche sicher tätig war.

Dass nicht nur legionare, sondern auch auxiliartruppen zu fuss und zu pferde, ja auch flottenmannschaften hier halfen, haben schon die umfassenden dedicationen aus der legatenzeit des Q. Acutius Nerva nr. 10.11..12, aber auch die sonderdedicationen der Asturier no. $18 \mathrm{~b}$ und 19 erwiesen. Derartige bescheidenere steine haben sich noch vorgefunden in no.

22. HERCVLI SAXANO SAGRY COH II. Brambach no. 658 liest am schluss: soh Il, Freudenberg no. 15: sacruso hii. Ich folge Jos. Klein, Bonner jabrb. 81, 115.

23. I. O. M. IVNON MARTI. HER SACRVM. C DOMITIVS RVFINVS. D. COH II C. R. PFD. ot COMmiLITONES VS. L. L. M. Bramb. no. 676. Catalog des Bonner mus. no. 26.

24. HeRCVLI SAXANO VeXellatio COHORTIS T. C. R. VSLM. Brambach no. 670. Freudenberg no. 10 liest: ICR statt TCR. Catalog d. Bonner mus. no. 24.

Die abkurzung $C R$ bedeutet eine cohors civium romanorum, wie sie oft noch durch den zusatz voluntariorum näher bestimmt wurde. In dieser zeit traten nämlich Italiker häufig, statt in

1) Schiller a. a. o. 1,607 ff. 
den schweren dienst der legion, als freiwillige in den leichteren der cohorten ein.1) Aher welcher legion diese römische burgercohorte und die zweite cohorte no. 22. 23 als hilfstruppen beigegeben waren, ist wol nicht mehr zu ermitteln.2)

Endlich hält auch die flotte, die classis Germanica, die wir schon nach no. 10-12 beteiligt fanden, ihre besondere verehrung für Hercules Saxanus nicht zurtick in no.

25. I. O. M. E herC. SAX VEXIL. CL. G. SVB CVRA RVFrI CALENI. TR E. IVL. L. IM . . S .. LM. Brambach no. 665. Freudenberg no. 20, und zwar unter führung eines $T R$ d. h. trierarcha, eines trieren- oder liburnencommandanten. ${ }^{3}$ ) Dazu mag man noch stellen die neuerdings im Brohltal gefundene ara:

26. HERCVLI. S F. NOBILI. CLASS. GER . . ET COMMILI . . . . V VB CV .... Bonner jahrb. 84, 85 ff.

Einzelne kaum nutzbare bruchstlicke wie 26 a : .. LASS... KE ET (?) COM. LITONES CLASSIS EIVSDEM . . LLM. Bonner jahrb. 84,62, in Tönnisstein beim Brobltal gefunden, kommen hier nicht in betracht.

Von sonderdedicationen der alae, der reiterei, die in no. 10-12 sich am Hercules-Saxanusdienst beteiligt, ist keine erhalten.

Durch die lange reihe dieser denkmäler no. 7-26 wird fur Vespasians und noch mehr fur Trajans zeit eine energische ausbeutung der Brohler steinbrttche bezeugt, aus der man wider auf eine bedeutende bautătigkeit beider kaiser am Niederrhein schliessen darf. Der zwischen ihnen regierende Domitian, der die reichsgrenze weiter rheinaufwärts namentlich von den Chatten bedroht sab, gentigte seiner baulust in Obergermanien mit dortigem material, indem or vorsorglich den wichtigen rechtsrheinischen limes in angriff nahm.4) Die Brohler briche lieferten ihre steine, wie es scheint, ausschliesslich Untergermanien. Hier aber hatte Vespasian nur. zu dringlichen anlass, baulich einzugreifen. Denn mit ausnahme von Mainz und dem entlegenen Windisch hatte Civilis sämmtliche winterlager der legionen, der reiterei und der cohorten, also insbesondere alle niedergerma-

1) Marquardt a. a. 0. $2^{2}, 467$.

2) Borghesi, Note sulle iscrizzione del Reno s. 12. Harster, Die nationen des Romerreichs in den heeren der kaiser s. 33.

3) Marquardt a. a. 0. $2^{2}, 505.509$.

4) Schiller a. a. 0. 1,517. Asbach, Westdeutsche zs. 3,1 ff. 
nischen, von grund aus zerstört.1) Schon am ende des jahres 70 liess Vespasian nach seinem. sieg sofort die winterlager von Bonn und Neuss notdurftig widerherstellen, aber erst in den nächsten jahren unternahm er den grundlichen neubau von Bonn, den ein wahrscheinlich in die mauer des neuen dortigen praetoriums gefugter stein mit der aufschrift: Vespasianus Imperator $X I V$, also aus dem jahre 75, bezeugt.2) Daher also die vorarbeiten der 8. und 21. legion in Brohl no. 7. 8. Am wege von der Brobl nach Bonn bei Oberwinter ist in basaltbrlichen widerum Hercules, wenn auch ohne beinamen, unter anderm auch auf einem tuffsteinaltar verehrt worden. ${ }^{3}$ ) Später stiftete in Bonn der legat L. Calpurnius Proclus demselben gotte einen stein aus dankbarkeit fur die vollendung eines lazareths, (p)eracto ope(r)e valetudina(rii), das futr die 1. legio Minervia bestimmt war.4) Gleichfalis in Bonn weihten der optio oder aufseher dieses valetudinarium und ein beneficiarius des legaten, der vielleicht mit dem vorgenannten die aufsicht teilte, einen stein dem Hercules Victor ${ }^{5}$ ) und widerum ein centurio der ersten Minervia dem Hercules Magusanus eine votivara aus Drachenfelser trachyt. ${ }^{6}$ ) Also auch in Bonn verehrte die Minervia wie im Brobltal no. 12 den Hercules und zwar auch als eine art Saxanus, nämlich als göttlichen helfer bei dem umfänglichen steinbau eines lazareths, wenn nicht, wie an andern orten, als Hercules Salutifer oder Salutaris. ${ }^{7}$ ) Und wie Hercules im Brohltal auch Invictus genannt wurde, no. $14 \mathrm{a}$, so heisst er in Bonn Victor. Der Bonner Herculescultus trăgt also dieselben hauptzlige wie der Brobler. Doch ob auch er schon der trajanischen zeit angehört, ist nicht zu bestimmen,

1) Tac. Hist. 4, 20.61.

2) Asbach, Westd. zs. 6, 231.

3) Bonner jahrb. 53, 141. Brambach no. 641-644. Catalog d. Bonner museums no. 27.

4) Bonner jahrb. 73, 63 .

5) Brambach no. 462, vgl. Marquardt a. a. o. 22, 549: beneficiarius legati agens c(uram) c(arceris) CIL. 3, 3412 und 8. 557: optiones valet (udinarii) CIL. 8, 2553. Bonner jahrb. 82, 53; vgl. Mommsen, Archäol. zeitung 1868 , s. 91 .

•) Bonner jahrb. 73, 74.

7) CIL. 3, 1572. 6, 237 vgl. 6, 338. 339. 
da die erste Minervia, wenn auch mit unterbrechungen, bis in Constantins zeit in Untergermanien verweilte.

Noch viel eifriger musste der Brohler tuffstein unter Trajan gebrochen werden, als dieser beschloss, das grosse, von Civilis zerstörte Vetera (bei Birten) durch ein neues, festes lager, die Castra Ulpia Trajana (bei Xanten), etwas weiter unterhalb zu ersetzen. Nun wurde auch in Brohl, wie frther in Mirebeau, eine zahlreiche baumannschaft aus verschiedenen truppenkörpern combiniert. Abteilungen jeder truppengattung mussten, unter dem commando eines centurio vereint, hand anlegen, wie die Acutiusinschriften no. 10-12 zeigen, legionare wie auxiliare und von diesen sowol die cohorten zu fuss, als auch die berittenen alae. Vielleicht dienten die pferde der reiterei dazu, die blöcke bis zum landungsplatz der classis germanica herab zu schleppen, die sie dann in ihre schiffe aufnahm und nach ihren bestimmungsörtern Bonn, Neuss, Nimwegen und namentlich nach castra Trajana hinabfuhrte. Das neue Trajanslager bezeugt das deutlich, denn seine umfassungsmauer mit ihrem durch turmbauten flankierten tor wurde aus so gewaltigem tuffsteinmaterial aufgefuhrt, dass noch in unserm jahrhundert ihrer ruine, als ob sio eine filiale des Brohltals wäre, das geschätzte baumaterial massenhaft entnommen wurde. ${ }^{1}$ ) Dass sich Trajans niedergermanische bautåtigkeit noch weiter erstreckte, beweist stromabwärts ein meilenstein an der heerstrasse nach Noviomagus bei Nimwegen ${ }^{2}$ ), stromaufwärts der wahrscheinlich unter ihm und Hadrian aus der Eifel nach Köln gefthrte aquaeduct. Auf verschiedene metalle schurften die Römer an mehreren orten des Mittelrheins; basalt brachen sie in Niedermendig, Mayen und Oberwinter, trachyt am Drachenfels und bei Berkum und tuff auch bei Kretz, Pleidt und Kruft bei Andernach. ${ }^{3}$ ) Aber das Brohltal ist als die mittelrheinische hauptrorratskammer zu betrachten, mit deren tuffsteinschätzen namentlich die kaiser Vespasian und Trajan die ausfibhrung ihrer grossen militärbauten am Niederrhein bestritten. Nach deren vollendung in der nachtrajanischen zeit scheint sie weniger in anspruch genommen worden zu sein. Schon die von Trajan errichtete

1) Bonner jahrb. 69, 70 .

2) Brambach no. 1927.

3) Bonner jahrb. $53-54$, s. 139. 
neue 30. legion, für die doch das neue trajanische lager bestimmt war, bat keine spur ibrer arbeit in den steinbrlichen binterlassen. Dass aber ausschliesslich untergermanische, nie obergermanische soldaten dieses doch zu Obergermanien gehörige tal ausbeuteten, darf nicht wundern. Hatte doch das gebirgige Obergermanien an gutem baumaterial überfluss, während das Brohler andrerseits sehr schwierig rheinaufwärts zu schaffen war.

Nach den hier vorgelegten urkunden haben also römische soldaten der 15. und der 16. niedergermanischen legion in der claudisch-neronischen epoche dem Hercules Saxanus im Brobltal die ersten uns bekannten denkmåler gesetzt. Bald darauf finden wir diesen gott in den kalksteinbrttehen von Norroy von soldaten einer obergermanischen, einer spanischen und einer moesischen legion und wider in Brohl von zwei obergermanischen und zwei spanischen legionen, von asturischen cohorten und italischen freiwilligen verehrt. War der procentsatz germanischen geblutes und geistes in den untergermanischen legionen schon vor Vespasians heeresreform viẹl zu gering, um sich gegentiber der tuberzahl fremder commilitonen und der uberlegenen cultur und stellung italischer officiere noch dazu bei solchen unter Germanen völlig ungebräucblichen stiftungen geltend machen zu können, so schwand dessen umfang und einfluss unter Vespasian und seinen nachfolgern in Germanien vollends zusammen, da diese kaiser, durch den Bataveraufstand belehrt, die in einer provinz ausgehobenen truppen in dieser provinz oder auch nur in deren năhe zu stationieren, sich wol hutteten. Germanische auxilien vollends scheinen seit Vespasian in den germanischen heeren nicht mehr zulässig. Der wahrscheinlich unter Vespasian begonnene ausschluss der Italiker traf aber zunächst nur diejenigen legionen, die während seiner regierung neu errichtet wurden, nicht die germanischen unsrer zeit.1) Viel stärker r'thrt sich augenscheinlich in diesen das keltische wesen, das wol durch zahlreichere mannschaft getragen und namentlich auf dem gebiete des cultus dem germanischen weit uberlegen war. Keltischer glaube konnte sich um so mehr bei diesen legionen behaupten, da sie auf einem nur dem namen nach germanischen,

1) Schiller a. a. o. 1,512. Mommsen im Hermes 19, 19. 214. 
in wirklichkeit fast durchweg keltischen boden ihre quartiere hatten. So erklärt sich, dass uns in den etwa drei dutzend stiftungen, die diese niedergermanischen legionen andern göttern als dem Hercules Saxanus weihen, keine spur eines germanischen gottesdienstes sichtbar wird, dagegen manche keltische gottheiten neben den römischen verehrung empfangen. Jene unglucklichen legionen no. 15 und 16 haben uns leider derartige zeugnisse nicht hinterlassen. Ein veteran der später ihr schicksal der auflösung teilenden 21. legion buldigt bei Vetera der Fortuna, einer ibrer soldaten dem Mercurius. $\left.{ }^{1}\right)$ Auch der 10. legion verdanken wir vielleicht einen Fortunastein. ${ }^{2}$ ) Die 6. aber votiert den matronis Rumanehabus, Axsinginehis und Afliabus, also anerkannt keltischen göttinnen ${ }^{3}$ ), aber auch der Deana (sic!), dem I. O. M. ohne oder mit andern römischen göttern und den Dis Manibus.. ${ }^{4}$ ) Diesen, wie dem I. 0. M.5) weiht auch die 22. legion mehrere steine, ausserdem der Diana und den Bivis Trivis Quadrivis und den ebenfalls zu den keltischen muttergöttinnen gehörenden Suleviabus ${ }^{6}$ ), endlich dem wahrscheinlich keltischen (?) gott Bacurdus, den auch die Classis germanica in Cöln verehrt. ${ }^{7}$ ) Die 1. legio Minervia weibt den Dis Manibus, der Diana, dem Apollo, dem I. O. M., dem Hercules Magusanus, endlich den Fortunis salutaribus, Aesculapio, Hyg(ieiae) ${ }^{8}$ ), aber am beliebtesten ist bei ihr der keltische matronencultus, insbesondere der der Aufaniae. ${ }^{9}$ ) Ein praefect dieser legion stellte aber auch im jabre 295 in Bonn einen verfallenen tempel des Mars militaris wider her, dessen ausser am Rhein

1) Brambach no. 210. 496.

2) Brambach no. 122.

8) Ihm, Bonner jahrb. 83, 1 ff., vgl. 161.

†) Bramb. no. 601. 336. 338. 81. 93. 500. Bonner jahrb. 81, 95. 83, 157. 160. 183.

5) Bramb. no. 199. 367. 909. 921. 1564.

6) Bramb. no. 328. 673. 1107. 1383.

7) Bramb. no. 386. 385, vgl. den iberischen namen Bacurius, aber anch den vielleicht thrakischen $B \varepsilon \lambda \sigma o v \tilde{d}$ os, Borghesi, Oeuvr. compl. 3, 274. Dumont im Arch. des missions scientif. 3, 3, 1876 s. 148 f. 182.

8) Bramb. no. 382. 332. 463. 481. 516. Bonner jahrb. 73, 74. 76. 47j 48, 161. 83, 162. Vgl. den Hygieiacultus in anderen provinzen des römischen westens, Roscher lexicon 1, 2786, vgl. Bonner jahrb. 57, 209.

9) Bramb. no. 329. 405. 541, vgl. 519? 560. 584. B. jb. 83,136. 156.164. 
nicht üblichen beinamen Th. Bergk wol mit recht als eine tubersetzung des keltischen kriegsgottes Caturix auffasste. ${ }^{1}$ ) Die 2. cohors civium Romanorum, deren erste (?) wir auch nach no. 24 im Brobltal trafen, widmet ganz römisch dem I. 0. M. Iun(oni). Marti Her(culi) in der nähe bei Andernach einen stein 2), die mit den asturischen cohorten von Hadrian nach Britannien gescbickte ala Asturum den Matribus oder den Matribus campestribus und ibrem Genius ${ }^{3}$ ); die Classis Germanica endlich weiht den Dis Manibus, dem I. O. M., der Diana, den Matribus suis und dem eben erwähnten Bacurdus.4) Der kreis der culte der betreffenden truppenkörper kann also ziemlich sicher umschrieben werden. Er enthält nichts germanisches. Jupiter, Diana, Fortuna, die dii Manes werden öfter, seltener Juno, Mars, Mercur, Apollo, Aesculap und Hygieia genannt. Mit ihnen teilen die verehrung der rätselhafte Bacurdus und ihre lieblingsgottheiten, die keltischen matres oder matronae. Wenn auch die phantastische ausstattung eines unter Acutius aufgestellten Herculesaltars (no. 10) unaufgeklärt bleibt, sie ist sicher nicht germanisch und schwerlich keltisch. Aber wie unrömisch sie auch ist, so muss man doch zugeben, dass nur Römer sie nach dem Rhein gebracht baben kōnnen. Auch haben wir schon gehört, dass im ubrigen der Hercules-Saxanus sich ganz naturlich aus dem römischen soldaten- und bauleben und der römischen Herculesvorstellung begreifen lässt, wåhrend jeglicher mangel eines keltischen zuges in den zahlreichen inschriften um so stärker gegen keltischen ursprung spricht, als diese bis hieher doch alle auf keltischem gebiete angefertigt sind. Offenbar ist nicht in Gallien, sondern in Italien der ausgangspunkt dieses Herculescultus zu suchen, und glucklicherweise stehen uns nun noch zwei Hercules-Saxanusdenkmäler zur verfügung, die sudlich von den Alpen entstanden. Freilich bringt uns der erste kaum einen neuen aufschluss, wol aber der zweite. Der erste Tridentiner trägt die inschrift:

27. HERCVLI SAXAN LVBIAMVS ENDRVR QVINTALLI VSL CILat. 5, 1, no. 5013. Freudenberg no. 24: Labiamus.

1) Bonner jahrb. 57, 21.

2) Bramb. no. 676.

8) Ihm, Bonner jahrb. 83, 157. 159. CIL. 7,510.

4) Bramb. no. 522. 355. 385. 684. 
Dieser stein fand sich in der Apollinariskirche zu Trient und hat vielleicht einst einem jener ergiebigen marmor- und gipsbritche angehört, die noch heute bei Trient ausgebeutet werden. $\mathrm{Ob}$ der dedicant mit seinem fremdartigen namen raetischer oder gallischer abkunft gewesen, kann ich nicht entscheiden. Tridentum war ursprünglich wol eine raetische stadt, die später in den besitz der keltischen Cenomanen gelangte. Aber im jahre 22 v. Chr. erbaute der legat Apulejus auf einem hügel uber ihr die Römerfestung Verruca.1)

Noch ein anderes denkmal des alpengebietes, das in den felsen Spitzhofen auf der Choralpe ob S. Georgen am Stein gehauene votivaltärchen mit der inschrift: S. SAXANO AVG. SAC ADIVTOR ET SECVNDINVS, wurde frther, so von Freudenberg no. 23 und Kaemmel, Anfänge d. deutschen lebens in Oesterreich 38, auf den Hercules Saxanus, wird jetzt aber richtiger auf Silvanus bezogen, CIL. 3, 2, no. 5093, mit dem sich jener allerdings oft berthrt. Die beinamen Sanctus und sogar Invictus sind beiden eigen. 1 )

Weit lehrreicher aber als die tridentinische widmung ist die tiburtinische, welche lautet:

28. HERCVLI SAXANO SACRVM SER SVLPICIVS TROPHIMVS AEDEM. ZOTHECAM. CVLINAM PECVNIA SVA A SOLO RESTITVIT IDEMQVE DEDICAVIT K. DECEMBR L. TVRPILIO. DEXTRO M. MAECIO. RVFO. COS. EVTYCHVS. SER. PeragendVim. CVRaVit. Freudenberg no. 25. ClL. 14, no. 3543.

Panvini und ihm folgend Freudenberg hatten diese inschrift ganz verkehrt in das jahr 225 gesetzt, wodurch sich offenbar auch noch $R$. Peter mit bestimmen liess, diesen Hercules für einen von germanischen soldaten vom Rhein an den Anio gebrachten gott zu halten. Aber nach $\mathrm{H}$. Dessau weist ihr ausser anderem schon die nennung von consules suffecti ein viel höheres alter zu, und höchst wahrscheinlich ist der eine consul suffectus M. Maecius Rufus identisch mit dem M. Maecius Rufus, der nach mtinzen unter Vespasian und Titus im jahre 79 proconsul von Bithynien war: ${ }^{3}$ ) Die bedeutung dieser neudatierung fur

1) CIL. 5, 1, s. 530. Revue celt. 11, 1890, 252. Egger, Geschichte Tirols 1, 29.

2) Preller, Röm. mythol. 23, 282. Peter bei Roscher a. a. o. 1, 2950. CIL. 7,451 .

3) CIL. 14, 375. Borghesi, Oeuv. 1, 511. 
unsere frage ist aber bisher trotzdem noch gar nicht erkannt. Die inschrift charakterisiert nicht nur mit fast grammatischer gründlichkeit das wort Saxanus, dessen zweites $\dot{a}$ sie wie die ubrigen langen $a$ in pecuniá, suá, $\dot{a}$, curávit mit einem apex versieht, als ein lateinisches - denn das oben leichthin ersonnene germanische wortgebild mutsste ein kurzes $a$ haben -, sondern sie zerstreut auch nach der glucklichen berichtigung ihres datums das dunkel, das immer noch uber der frage nach der engeren heimat unseres Hercules lagert. Sie bezeugt nicht nur einen italischen Hercules Saxanus vor den toren Roms in der vespasianischen zeit und erweist sich als fast gleichaltrig mit den altesten Hercules-Saxanusurkunden des nordens, sondern erhebt sich auch uber diese als die älteste unserer sämmtlichen bezeugungen des gottes durch den ausdruck aedem etc. a solo restituit. Hercules Saxanus batte also bei Tibur einen älteren, unter Vespasians regierung bereits verfallenen tempel. Dazu stimmt treff lich eine notiz Strabos c. 238, der um Christi geburt unterhalb Tibur grossartige steinbriche kannte, an denen der damals schiffbare Anio vorbeifloss, um ihre ausgehauenen blöcke zu den baustätten der hauptstadt hinabzutragen. Noch heute sind sie als cave di Travertino bekannt. Der travertin, der am rande der vulcanischen Campagna abgelagerte 'Tiburtiner kalkstein, fieng wahrscheinlich erst nach Karthagos und Korinths zerstörung an, die abrigen materiale der grabmäler, altäre und votivsteine in Rom zu verdrängen und wurde zu grösseren bauten zuerst in der zeit des jungeren Catulus zwischen 7860 v. Chr. ausserst sparsam und erst seit Augustus ausgiebiger benutzt.1) Dieser richtete aus tiburtinischen quadern zwar nicht, wie Burckhardt angibt, auf seinem forum den prachtvollen tempel des Mars Ultor auf, fugte aber travertinemposten in die schutzmauer dieses forums ein.2) Der āltere Saxanustempel von Tibur kann also schon unter den ersten kaisern gestanden haben, thertraf wol jedenfalls an alter die fruhesten Brohler Saxanussteine. Wie Vespasian fur seine bauten in Bonn den nächsten von Hercules Saxanus beschutzten steinbruch der Brohl

1) Jordan, Topogr. d. stadt Rom 1, 1, 6 ff. 1, 2,141 ff.

2) Monum. Ancyr. 4, 21. Jordan a. a. 0. 1, 2, 446. Burckhardt, Cicerone $1^{3}, 21$. Preller, Röm. myth. $1^{3}, 368$. 
benutzte, beutete er für seine hauptstädtischen werke, jedenfalls für das aus travertin aufgefuhrte colosseum, den durch die wasserstrasse des Anio so bequem mit Rom verbundenen steinbruch von Tibur aus, der unter den schutz desselben gottes schon früher gestellt.war. Das flavische amphitheater hatte später ein ähnliches schicksal wie die castra Trajana am Niederrhein. Diese wurden zu einer tuffsteingrube moderner bauleute (o.s.124), jenes zu einem steinbruch, aus dessen material die vornehmsten paläste der renaissance in Rom emporstiegen.1) Der von der tiburtinischen inschrift erwähnte Servius Trophinus mag einer der steinbruchsprocuratoren gewesen sein, wie sie wenigstens seit Trajan nachweisbar sind, und der servus Eutychus ein betriebsdirector oder architekt,. der damals ein sclave zu sein pflegte. Jener erachtete ${ }^{2}$ ) den neubau des verfallenen HerculesSaxanustempels für angemessen, um des gottes huld für die saure und gefahrvolle arbeit seiner leute zu gewinnen.

Viel berthmter aber war Tibur, die urbs Herculi sacra (Sueton. Calig. 8), durch einen andern Herculestempel, den des Hercules Victor oder Invictus, dessen reste in den angeblichen ruinen der villa des Maecenas widergefunden sind. Den kriegerischen charakter des gottes beweist ausser seinem beinamen der umstand, dass die Salier, die in Rom ausschliesslich priester- des Mars waren, in Tibur nicht nur diesen, sondern auch den Hercules bedienten. In Tibur hatte Hercules einst dem Jupiter einen altar gestiftet, wahrscheinlich nach einem ähnlichen abenteuer, wie er es in der römischen Cacussage bestanden hatte.3) Ein Tiburtiner Octavius Herrenus oder Hersennius sollte den altertumlichen rundtempel des Hercules Victor in Rom gegründet baben, und so viel ist jedenfalls an dieser geschichte wahr, dass die Herculesdienste der beiden nachbarstädte Tibur und Rom aufs engste mit einander verwant waren. Auch der römische Hercules stiftete dem Jupiter und zwar dem Inventor einen altar, die weltbekannte ara maxima auf dem forum Boarium, und fubrte

1) Friedländer, Darstellungen a. d. sittengesch. Roms $2^{1}, 261$. Bädeker, Italien $2^{5}, 221.347$.

2) Marquardt ${ }^{2}$ a. o. 2, 264.

8) Dessau CIL. 14, 367. Preller, Röm. myth. 18, 352. Peter a. a. o. 1,3003 . 
neben seinem älteren beinamen Victor den des Invictus.1) Neben diesen ist der name Saxanus in Rom zwar nicht bezeugt, jedoch scheint der Hercules Victor mit der Bona dea Subsaxana im innigsten bunde gestanden zu haben; denn seine ara maxima sammt jenem rundtempel lag unmittelbar am fusse des Aventinus, unter dessen saxum die Bona Dea hauste, die auch als tochter des Faunus für eine gemalin des Hercules galt.2) Wie es scheint, standen auch die culte beider in einem inneren zusammenhang, nach welchem die frauen von den opferschmäusen des Hercules und umgekehrt die männer von denen der Bona Dea ausgeschlossen waren. ${ }^{3}$ ) Doch gentigt mir hier der hinweis auf den uralten hauptalter des Hercules, die ara maxima auf dem forum Boarium neben seinem rundtempel. Vor diesem opferte alljälrlich der stadtpraetor in gegenwart der burgerschaft eine kuh und wurden bis tief in die kaiserzeit schwelgerische festmahle gehalten. Mit der löwenhaut haupt und nacken bedeckt, stand der gott in seinem heiligtum, wo man noch seine keule und seinen mächtigen humpen zeigte. Bei den triumphztigen emptieng er, mit dem triumphalkleid geschmlickt, den zehnten der beute, den der siegreiche feldherr vor ihm niederlegte.4) Von hier aus drang sein ruhm durch das ganze römische heer. Grade diesen römisch-tiburtinischen Hercules in seiner kriegerischen und gewerblichen doppeleigenschaft finden wir am Mittelrhein in genauerer entsprechung als irgendwo sonst im römischen reiche wider. Der wechselnde gebrauch seiner beiden beinamen Victor und Invictus kommt ausserbalb Roms und Tiburs ${ }^{5}$ ) kaum innerbalb eines andern engeren bezirkes des weiten reiches vor, als im mittelrheinischen, wo wir einen Hercules Victor in Bonn (s. 123) und einen Her-

1) Peter a. a. 0. 1, $2907 \mathrm{ff}$. 2923. Jordan, Topogr. der stadt Rom $1,2,482$.

2) Peter a. a. o. 1, 2291.

3) Preller, Röm. myth. $2^{3}, 283$. Später ruft der mann den Hercules, die frau die Junones an. Ihm, Bonner jb. 83, 77. 117.

4) Peter a. a. 0. 1, 2907. 2916 ff. Jordan, Topogr. d. stadt Rom 1, 2, 477 ff. 482.

5) Dessau a. a. o. Peter a. a. o. 1, 2906. 2923. Hüchstens könnte man den Hercules Invictus in Neapel und den Hercules Victor in Aquino anfübren CIL. 10, 1478. 5386. 
cules Invictus im Brohltal (no. 14 a) trafen, und vollends schmücken alle drei namen: Victor, Invictus und Saxanus ihn nur in Tibur und am Mittelrhein. In Brohl und in Norroy wurde seine verehrung aufs innigste mit der seines vaters Jupiter verknüpft (no. 3. 5. 11. 14. 15. 23), was widerum grade in Rom und Tibur durch den charakteristischen mythus bezeugt wird, nach welchem Hercules einen Jupiteraltar stiftete.

Bildliche darstellungen des Hercules vom Brohl- und Moseltal sind uns leider nicht erhalten. Doch jene zwar aus Griechenland uberkommene, aber stadtrömische auffassung scheint auch die Herculesbilder in Germanien beherscht zu haben. Denn meistens ist auf den obergermanischen viergöttersteinen der gott mit der löwenhaut versehen und hält in der rechten die keule, in der linken allerdings in der regel die Hesperidenăpfel, aber auch wol eine schale, einen krug, einen becher. $\left.{ }^{1}\right)$ Auch ein Nehalenniastein der insel Walcheren stellt ihn dar sitzend in der löwenhaut mit einem skyphos in der hand.2) Endlich findet sich auch die verbindung des Hercules mit Sol und Luna, die wir aus der ausstattung eines seiner Brohler altäre (no. 10) erschliessen zu dưrfen glaubten, in den weihinschriften der in Rom casernierten Equites singulares wider (s. 118).

Die Hercules-Saxanusfrage scheint mir jetzt im wesentlichen gelöst. Der traum von seinem Germanen- oder Keltentum ist zerronnen und klar tritt sein römischer charakter heraus. Mogen seine culte dem Etsch-, Mosel- oder Rheintale angehören, sie sind alle von Rom und dessen nåchsten umkreis, vom herzen des reichs, ausgegangen, wo der Griechenheros durch den staat frluh in den kreis der heimischen götter einbezogen worden war. In Tibur wurde er schon vor Vespasian verehrt, vor und während dessen regierungszeit auch die ältesten steine der Brohl gesetzt sind. Mit den baulichen aufgaben dieses kaisers und Trajans nahm der Hercules-Saxanuscult einen neuen aufschwung in den steinbruchen am Anio, an der Mosel und am Rhein und verkntupfte sich mit denkwturdigen

1) Westdeutsche zs. 10, 305 ff. 145. 147.

2) Janssen, De romeinsche beelden en gedenksteenen van Zeeland 1845 , s. 41 tafel 8 a b c. 
ereignissen der geschichte. Was aus ihm nach Trajans zeit geworden ist, wissen wir nicht. Von dem vornehmen und kriegerischen heros Hercules Victor oder Invictus abgezweigt, steht der Saxanus etwa in der mitte zwischen diesem und dem friedlichen Hercules tutor, dem huter des hofs und der heerden, dem Hercules rusticus, agrestis, campanus, dem freund der bauern und ihrer feldarbeit, als der behuter römischer steinmetzen und steinbrecher, blurgerlicher wie soldatischer, dem sie ein monument des dankes setzten, wenn sie.ihr schweres werk im felsgestein vollendet hatten.

FREIBURG I. B., jan. 1893.

E. H. MEYER. 